\title{
Screening of bactericidal activity of selected Plumbago species against bacterial pathogens
}

\begin{abstract}
The present study was undertaken to determine the antibacterial potential of Plumbago species viz., Plumbago zeylanica Linn., Plumbago auriculata Lam. and Plumbago rosea Linn. collected from various localities of South India. For the bio-efficacy studies six different extracts of Plumbago species with various concentrations viz., 20, 40, 60, 80 and $100 \mu \mathrm{g} / \mathrm{ml}$ against gram positive and gram negative bacterial pathogens viz., Staphylococcus aureus (MTCC 737), Streptococcus pyogenes (MTCC 1928), Bacillus subtilis (MTCC 441), Klebsiella pneumoniae (MTCC 109), Morganella morganii (MTCC 662) and Pseudomonas aeruginosa (MTCC 1688) using well diffusion method. Among the eighteen different extracts of three different Plumbago species, highest frequency of antibacterial activity (54\%) was recorded in $P$. rosea. The antibacterial activity of various extracts in different concentration of the selected Plumbago species are as follows: P. rosea $(54 \%)>P$. zeylanica $(49 \%)>P$. auriculata $(40 \%)$. The ethanolic extract of all the Plumbago species revealed superior bactericidal activity compared to other tested extracts. The ethanolic extracts of $P$. zeylanica, $P$. auriculata and $P$. rosea showed $91 \%, 50 \%$ and $99 \%$ of activity against Gram positive pathogens and $66 \%, 26 \%$ and $89 \%$ of activity against Gram negative pathogens. The bioactive compound plumbagin and extract of aerial parts of Plumbago species show a wide spectrum of antibacterial activity. The compound shows promise as a new drug for various bacterial infectious diseases. Hence, this study offers a base of using Plumbago species as herbal alternative for the synthesis of antimicrobial agents.
\end{abstract}

Volume 2 Issue 6 - 2015

\section{Renisheya Joy Jeba Malar Tharmaraj, Johnson Marimuthu Antonysamy \\ Department of Botany, Centre for Plant Biotechnology, India}

Correspondence: Johnson Marimuthu Antonysamy, Centre for Plant Biotechnology, Department of Botany, St. Xavier's College (Autonomous), Palayamkottai, Tamil Nadu, India, Tel +979786924334, Fax +914622561765, Email ptcjohnson@gmail.com

Received: July 28, 2015 | Published: December 03, 2015

Keywords: bactericidal, Plumbago species, antibacterial, extracts

\section{Introduction}

Plants and plant derived metabolites are believed as rich sources of antimicrobial agents. A wide range of plants and their parts are used for their medicinal properties by local communities and folklore healers. Plants possess varieties of secondary metabolites with antimicrobial properties. The studies on plant derived polyphenolic compounds confirmed the antimicrobial, antioxidant, anticancer and apoptosis inducing properties of the plants and supplemented effective usage of medicinal herbs against microorganisms. ${ }^{1,2}$ With the impact and less side effects, the herbal medicine is practiced as complementary and alternative medicine day by day in developing countries. ${ }^{3,4}$ Plants and plant derived products are the cheapest, easily avialable and safer alternative sources of antimicrobials. ${ }^{5-7}$ The phytochemical and pharmacological studies on the roots of $P$. zeylanica confirmed antiplasmodial, ${ }^{8}$ antimicrobial, ${ }^{9}$ antifungal, ${ }^{10}$ anti inflammatory and anticancer, ${ }^{11}$ antihyperglycemic, ${ }^{12}$ hypolipidaemic and anti atherosclerotic, ${ }^{13}$ properties of the plant.

The ethnobotanical knowledge and biological studies on the aerial parts or roots of $P$. auriculata suggested that root and aerial parts of $P$. auriculata are employed to control black water fever, ${ }^{7}$ plant are used as anti feedant ${ }^{14}$ and antifungal agents to control spore germination of Macrophomina phaseolina. ${ }^{15}$ The ethanolic leaf extract of $P$. rosea is active against herpus simplex virus type I. The root of $P$. rosea was used to treat digestive problems, dyspepsia, colic cough and bronchitis. ${ }^{16}$ Ibrahim et al., ${ }^{17}$ studied the antibacterial potentials of $P$. indica. Devi et al., ${ }^{18}$ screened the antibacterial efficacies of $P$. zeylanica leaf extracts. Vishnukanta \& Rana ${ }^{19}$ studied the anti convulasant activity Plumbago zeylanica, Jeyachandran et al. ${ }^{20}$ evaluated the antibacterial activity of plumbagin and root extracts of Plumbago zeylanica, Rahman \& Anwar ${ }^{21}$ screened the antimicrobial activity of Plumbago zeylanica root crude extract. Parekh \& $\mathrm{Chanda}^{22}$ studied the antibacterial potentials of $P$. zeylanica. Tharmaraj \& Antonysamy ${ }^{23}$ screened the antibacterial efficacy of $P$. rosea from Chankanachari, Kerala. Most of the studies were focused on the antimicrobial potentials of $P$. zeylanica roots only; very few reports are available on aerial parts of the selected Plumbago species of Tamil Nadu. There is no report on the antimicrobial actvities of Plumbago auriculata. There is no report on the antimicrobial actvities of Plumbago auriculata. To supplement the previous observations, an attempt has been made to reveal the bactericidal activity of three selected Plumbago species viz., Plumbago zeylanica Linn., Plumbago auriculata Lam. and Plumbago rosea Linn. aerial parts.

\section{Materials and methods}

\section{Preparation of extracts}

For bioefficacy studies, the aerial parts of Plumbago zeylanica Linn., Plumbago auriculata Lam. and Plumbago rosea Linn. were collected from Papanasam (Tamil Nadu), Tenkasi (Tamil Nadu) and Dana (Tamil Nadu) respectively. The collected Plumbago species were washed thoroughly with tap water followed by distilled water. The washed Plumbago species were blotted on the blotting paper and spread out at room temperature in shade to remove the excess water contents. The shade dried plant samples were ground to fine powder using mechanical grinder. The powdered samples were stored at $4^{\circ} \mathrm{C}$ for further use.

The dried and powdered aerial parts of Plumbago species were extracted successively with $30 \mathrm{~g}$ of plant powder and $180 \mathrm{ml}$ of petroleum ether, chloroform, acetone, ethyl acetate, ethanol and 
water using soxhlet extractor for $8 \mathrm{hr}$ at a temperature not exceeding the boiling point of the solvent. The extracts were filtered using Whattman filter paper (No.1) and then concentrated in vacuum at $40^{\circ} \mathrm{C}$ using rotary evaporator. The residues obtained were stored in a freezer until further tests. One gram of plant extracts were diluted with $1 \mathrm{ml}$ of the respective solvents. They were used as stock solutions for the atibacterial assay.

\section{Preparation of the test organisms}

Staphylococcus aureus (MTCC 737), Streptococcus pyogenes (MTCC 1928), Bacillus subtilis (MTCC 441), Klebsiella pneumoniae (MTCC 109), Morganella morganii (MTCC 662) and Pseudomonas aeruginosa (MTCC 1688) were commercially purchased from Institute of Microbial Technology, Chandigarh, India. Stock cultures of different bacteria were grown in nutrient broth at $30{ }^{\circ} \mathrm{C}$ and were sub-cultured and maintained in nutrient broth at $4^{\circ} \mathrm{C}$. Before swabbing, each culture was diluted $(1: 10)$ with fresh sterile nutrient broth.

\section{Antibacterial assay}

The antibacterial activity was determined by the agar well diffusion method Parekh \& Chanda. ${ }^{24}$ A suspension of the culture organism was swabbed above the solidified Muller Hinton agar medium. Wells were made using sterile cork borer under aseptic condition. One millgram of plant extracts were diluted with $1 \mathrm{ml}$ of the respective solvents. They were used as stock solutions for the atibacterial assay. From the stock the plant extracts $(1 \mathrm{mg} / \mathrm{ml})$ various concentrations viz., 20, 40, 60,80 and $100 \mu \mathrm{g} / \mathrm{ml}$ were prepared and inoculated in the wells. The antibiotic amikacin $(30 \mu \mathrm{g} / \mathrm{disc})$ was used as a standard to compare its effect on test organisms with the plant extracts. The plates were kept at room temperature for $2 \mathrm{~h}$ to allow diffusion of the test solution into the agar then they were incubated for $24 \mathrm{~h}$ at $37^{\circ} \mathrm{C}$. After the incubation period, the plates were observed and zone of inhibition was measured $(\mathrm{mm})$ and the activities were recorded.

\section{Results}

Among the three species studied, highest frequency of antibacterial activity (54\%) was recorded in $P$. rosea. The range of antibacterial activity of various extracts of Plumbago species are as follows: $P$. rosea $(54 \%)>P$. zeylanica $(49 \%)>P$. auriculata $(40 \%)$. Major antibacterial activities were observed predominantly in ethanolic extracts $(85 \%)$ of $P$. zeylanica. However the range of the inhibition zone varied with test organisms based on different concentration of extracts. Highest antibacterial activity was observed in ethanolic extract at $100 \mu \mathrm{g} / \mathrm{ml}$ followed by petroleum ether and chloroform extract. There was minor difference on the size of the inhibition zone between ethanolic and other extracts of $P$. zeylanica. The range of inhibitory activity was less at lower concentration of extracts (Table 1). Ethanolic extracts of P. zeylanica was active against all the selected bacterial pathogens except $B$. subtilis which represents no zone of inhibition at $20-40 \mu \mathrm{g} / \mathrm{ml}$ concentration. Compared to standard amikacin $(30 \mu \mathrm{g}), 100 \mu \mathrm{g} / \mathrm{ml}$ of ethanolic extract showed more antibacterial $(90 \%$ and $5 \%)$ activity against $S$. pyogenes and $K$. pneumonia respectively (Table 1$)$. The ethanolic extracts of $P$. zeylanica showed higher antibacterial activity against pathogens $K$. pneumonia and $S$. pyogenes. The petroleum ether extract of $P$. zeylanica also expressed maximum zone of inhibition $(20 \pm 0.5 \mathrm{~mm})$ against $S$. pyogenes. The acetone and aqueous extracts of $P$. zeylanica failed to show the antibacterial activity against $S$. aureus. Similar to that, the petroleum ether, acetone and aqueous extracts of $P$. zeylanica were also unsuccessful against $B$. subtilis. The antibacterial activity of $P$. zeylanica extracts at different concentrations are arranged as follows: ethanolic extracts $(85 \%)>$ petroleum ether $(69 \%)>$ chloroform $(62 \%)>$ acetone $(53 \%)>$ ethyl acetate $(34 \%)>$ aqueous extract $(31 \%)$. Ethanolic extracts of $P$. zeylanica showed $91 \%$ percentage of activity against Gram positive pathogens and $66 \%$ of activity against Gram negative pathogens.

Table I Antibacterial activity of Plumbago zeylanica

\section{Zone of inhibition in $\mathrm{mm}$}

\begin{tabular}{|c|c|c|c|c|c|c|c|c|}
\hline \multirow{2}{*}{ Pathogens } & \multirow{2}{*}{ Extracts Conc. in $\mu g$} & & & & & & & \\
\hline & & $\mathbf{P}$ & C & A & EA & E & $A Q$ & Amikacin $30 \mu g$ \\
\hline \multirow{5}{*}{ S. aureus } & 20 & $10 \pm 0.2$ & $6 \pm 0.3$ & nil & nil & $6 \pm 0.3$ & nil & \multirow{5}{*}{24} \\
\hline & 40 & $15 \pm 0.3$ & $9 \pm 0.3$ & nil & nil & $10 \pm 0.5$ & nil & \\
\hline & 60 & $17 \pm 0.3$ & $10 \pm 0.5$ & nil & $3 \pm 0.3$ & $15 \pm 0.3$ & nil & \\
\hline & 80 & $18 \pm 0.4$ & $13 \pm 0.5$ & nil & $4 \pm 0.3$ & $18 \pm 0.5$ & nil & \\
\hline & 100 & $19 \pm 0.3$ & $16 \pm 0.5$ & nil & $6 \pm 0.5$ & $20 \pm 0.5$ & nil & \\
\hline \multirow{5}{*}{ B. subtilis } & 20 & nil & $5 \pm 0.3$ & nil & $6 \pm 0.5$ & nil & nil & \multirow{5}{*}{25} \\
\hline & 40 & nil & $8 \pm 0.5$ & nil & $8 \pm 0.3$ & nil & nil & \\
\hline & 60 & nil & $10 \pm 0.5$ & nil & $10 \pm 0.4$ & $5 \pm 0.3$ & nil & \\
\hline & 80 & nil & $12 \pm 0.3$ & nil & $12 \pm 0.3$ & $7 \pm 0.5$ & nil & \\
\hline & 100 & nil & $13 \pm 0.5$ & nil & $16 \pm 0.3$ & $9 \pm 0.3$ & nil & \\
\hline \multirow{5}{*}{ S. pyogenes } & 20 & $14 \pm 0.3$ & nil & $5 \pm 0.3$ & nil & $15 \pm 0.3$ & nil & \multirow{5}{*}{11} \\
\hline & 40 & $16 \pm 0.3$ & nil & $10 \pm 0.3$ & nil & $18 \pm 0.5$ & nil & \\
\hline & 60 & $17 \pm 0.3$ & nil & $12 \pm 0.5$ & nil & $19 \pm 0.3$ & $3 \pm 0.5$ & \\
\hline & 80 & $19 \pm 0.5$ & $5 \pm 0.3$ & $14 \pm 0.3$ & nil & $20 \pm 0.5$ & $4 \pm 0.5$ & \\
\hline & 100 & $21 \pm 0.5$ & $7 \pm 0.5$ & $18 \pm 0.5$ & nil & $21 \pm 0.3$ & $6 \pm 0.5$ & \\
\hline
\end{tabular}


Table continued

\begin{tabular}{|c|c|c|c|c|c|c|c|c|}
\hline \multirow{2}{*}{ Pathogens } & \multicolumn{8}{|c|}{ Zone of inhibition in $\mathrm{mm}$} \\
\hline & Extracts Conc. in $\mu g$ & $\mathbf{P}$ & C & A & EA & $\mathbf{E}$ & AQ & Amikacin $30 \mu g$ \\
\hline \multirow{5}{*}{ K. pneumoniae } & 20 & $3 \pm 0.3$ & $5 \pm 0.3$ & $3 \pm 0.3$ & $4 \pm 0.3$ & $17 \pm 0.3$ & nil & \multirow{5}{*}{21} \\
\hline & 40 & $4 \pm 0.3$ & $10 \pm 0.3$ & $6 \pm 0.3$ & $6 \pm 0.5$ & $19 \pm 0.3$ & nil & \\
\hline & 60 & $6 \pm 0.3$ & $12 \pm 0.5$ & $8 \pm 0.3$ & $7 \pm 0.5$ & $20 \pm 0.5$ & $3 \pm 0.5$ & \\
\hline & 80 & $8 \pm 0.3$ & $14 \pm 0.3$ & $10 \pm 0.3$ & $10 \pm 0.3$ & 21 & $5 \pm 0.5$ & \\
\hline & 100 & $10 \pm 0.3$ & $18 \pm 0.5$ & $12 \pm 0.5$ & $12 \pm 0.5$ & $22 \pm 0.3$ & $7 \pm 0.5$ & \\
\hline \multirow{5}{*}{ M. morganii } & 20 & nil & $12 \pm 0.3$ & nil & nil & $8 \pm 0.3$ & $8 \pm 0.2$ & \multirow{5}{*}{20} \\
\hline & 40 & nil & $16 \pm 0.3$ & $5 \pm 0.5$ & nil & $12 \pm 0.3$ & $9 \pm 0.2$ & \\
\hline & 60 & nil & $17 \pm 0.5$ & $8 \pm 0.5$ & nil & $15 \pm 0.5$ & $10 \pm 0.2$ & \\
\hline & 80 & $3 \pm 0.3$ & $19 \pm 0.5$ & $9 \pm 0.2$ & nil & $16 \pm 0.5$ & $12 \pm 0.2$ & \\
\hline & 100 & $6 \pm 0.5$ & $21 \pm 0.5$ & $I I \pm 0.3$ & nil & $19 \pm 0.5$ & $14 \pm 0.5$ & \\
\hline \multirow{5}{*}{ P. aeruginosa } & 20 & $7 \pm 0.3$ & nil & nil & $3 \pm 0.5$ & $2 \pm 0.3$ & nil & \multirow{5}{*}{24} \\
\hline & 40 & $9 \pm 0.5$ & nil & $2 \pm 0.3$ & $4 \pm 0.3$ & $4 \pm 0.3+$ & nil & \\
\hline & 60 & $1 I \pm 0.5$ & nil & $5 \pm 0.5$ & $7 \pm 0.5$ & $7 \pm 0.5$ & nil & \\
\hline & 80 & $12 \pm 0.5$ & nil & $8 \pm 0.3$ & $8 \pm 0.5$ & $10 \pm 0.3$ & nil & \\
\hline & 100 & $16 \pm 0.5$ & nil & $10 \pm 0.3$ & $10 \pm 0.3$ & $12 \pm 0.3$ & nil & \\
\hline
\end{tabular}

Note: P, Petroleum ether; C, Chloroform;A, Acetone; EA, Ethylacetate; E, Ethanol;AQ,Water

Similar to $P$. zeylanica, highest activity was observed in ethanolic extracts $(70 \%)$ of $P$. auriculata followed by petroleum ether and chloroform extracts. There was distinguished difference on the size of the inhibition zone between ethanolic and other extracts of
P.auriculata. The range of inhibition was directly coincided with the concentrations of extracts tested, less inhibition was observed at lower concentration of extracts and maximum inhibition was obtained in higher concentrations (Table 2).

Table 2 Antibacterial activity of Plumbago auriculata

\begin{tabular}{|c|c|c|c|c|c|c|c|c|}
\hline \multirow{2}{*}{ Pathogens } & \multicolumn{8}{|c|}{ Zone of inhibition in $\mathrm{mm}$} \\
\hline & Extracts Conc. in $\mu g$ & $\mathbf{P}$ & C & A & EA & $\mathbf{E}$ & $\mathbf{A Q}$ & Amikacin $30 \mu g$ \\
\hline \multirow{5}{*}{ S. aureus } & 20 & $4 \pm 0.3$ & $2 \pm 0.3$ & nil & $4 \pm 0.3$ & nil & nil & \multirow{5}{*}{24} \\
\hline & 40 & $6 \pm 0.3$ & $4 \pm 0.3$ & nil & $7 \pm 0.3$ & nil & nil & \\
\hline & 60 & $8 \pm 0.3$ & $7 \pm 0.7$ & nil & $8 \pm 0.3$ & nil & nil & \\
\hline & 80 & $10 \pm 0.3$ & $9 \pm 0.5$ & nil & $11 \pm 0.3$ & nil & $3 \pm 0.5$ & \\
\hline & 100 & $12 \pm 0.3$ & $11 \pm 0.3$ & nil & $13 \pm 0.3$ & nil & $4 \pm 0.3$ & \\
\hline \multirow{5}{*}{ B. subtilis } & 20 & $6 \pm 0.3$ & nil & $\mathrm{I} \pm 0.3$ & $2 \pm 0.3$ & $4 \pm 0.3$ & nil & \multirow{5}{*}{25} \\
\hline & 40 & $8 \pm 0.4$ & nil & $2 \pm 0.3$ & $4 \pm 0.3$ & $6 \pm 0.3$ & nil & \\
\hline & 60 & $10 \pm 0.3$ & $6 \pm 0.5$ & $4 \pm 0.3$ & $5 \pm 0.3$ & $7 \pm 0.5$ & $I \pm 0.3$ & \\
\hline & 80 & $13 \pm 0.5$ & $9 \pm 0.5$ & $6 \pm 0.3$ & $7 \pm 0.2$ & $13 \pm 0.3$ & $4 \pm 0.3$ & \\
\hline & 100 & $15 \pm 0.3$ & $10 \pm 0.3$ & $8 \pm 0.3$ & $10 \pm 0.3$ & $16 \pm 0.3$ & $6 \pm 0.3$ & \\
\hline \multirow{5}{*}{ S. pyogenes } & 20 & nil & nil & $4 \pm 0.3$ & $10 \pm 0.5$ & $4 \pm 0.3$ & nil & \multirow{5}{*}{ II } \\
\hline & 40 & nil & nil & $6 \pm 0.3$ & $13 \pm 0.3$ & $6 \pm 0.5$ & nil & \\
\hline & 60 & nil & nil & $7 \pm 0.3$ & $14 \pm 0.3$ & $7 \pm 0.5$ & $3 \pm 0.5$ & \\
\hline & 80 & nil & nil & $10 \pm 0.3$ & $16 \pm 0.3$ & $9 \pm 0.3$ & $4 \pm 0.5$ & \\
\hline & 100 & nil & nil & $12 \pm 0.3$ & $18 \pm 0.3$ & $13 \pm 0.5$ & $6 \pm 0.5$ & \\
\hline
\end{tabular}




\begin{tabular}{|c|c|c|c|c|c|c|c|c|}
\hline \multirow{2}{*}{ Pathogens } & \multicolumn{8}{|c|}{ Zone of inhibition in $\mathrm{mm}$} \\
\hline & Extracts Conc. in $\mu g$ & $\mathbf{P}$ & C & A & EA & $\mathbf{E}$ & AQ & Amikacin $30 \mu g$ \\
\hline \multirow{5}{*}{ K. pneumoniae } & 20 & $7 \pm 0.5$ & $4 \pm 0.3$ & nil & $4 \pm 0.3$ & $13 \pm 0.5$ & nil & \multirow{5}{*}{21} \\
\hline & 40 & $9 \pm 0.7$ & $6 \pm 0.3$ & nil & $6 \pm 0.5$ & $15 \pm 0.3$ & nil & \\
\hline & 60 & $14 \pm 0.3$ & $8 \pm 0.3$ & $3 \pm 0.3$ & $7 \pm 0.5$ & $17 \pm 0.3$ & $3 \pm 0.5$ & \\
\hline & 80 & $18 \pm 0.3$ & $I I \pm 0.3$ & $6 \pm 0.3$ & $10 \pm 0.3$ & $19 \pm 0.3$ & $5 \pm 0.5$ & \\
\hline & 100 & $20 \pm 0.5$ & $14 \pm 0.3$ & $7 \pm 0.3$ & $13 \pm 0.3$ & $23 \pm 0.3$ & $7 \pm 0.5$ & \\
\hline \multirow{5}{*}{ M. morganii } & 20 & $3 \pm 0.5$ & $2 \pm 0.3$ & nil & nil & nil & nil & \multirow{5}{*}{20} \\
\hline & 40 & $5 \pm 0.5$ & $4 \pm 0.3$ & nil & nil & $2 \pm 0.3$ & nil & \\
\hline & 60 & $8 \pm 0.3$ & $6 \pm 0.5$ & nil & nil & $4 \pm 0.3$ & nil & \\
\hline & 80 & $10 \pm 0.3$ & $8 \pm 0.3$ & $3 \pm 0.3$ & nil & $6 \pm 0.3$ & nil & \\
\hline & 100 & $14 \pm 0.5$ & $1 \mathrm{II} \pm 0.5$ & $4 \pm 0.3$ & nil & $8 \pm 0.3$ & nil & \\
\hline \multirow{5}{*}{ P. aeruginosa } & 20 & $6 \pm 0.3$ & nil & $I I \pm 0.3$ & nil & $2 \pm 0.3$ & $2 \pm 0.3$ & \multirow{5}{*}{24} \\
\hline & 40 & $9 \pm 0.3$ & nil & $14 \pm 0.3$ & nil & $3 \pm 0.3$ & $4 \pm 0.3$ & \\
\hline & 60 & $1 \mathrm{II} \pm 0.3$ & nil & $16 \pm 0.3$ & nil & $5 \pm 0.5$ & $7 \pm 0.5$ & \\
\hline & 80 & $14 \pm 0.3$ & nil & $18 \pm 0.3$ & nil & $7 \pm 0.3$ & $10 \pm 0.3$ & \\
\hline & 100 & $16 \pm 0.3$ & nil & $20 \pm 0.3$ & nil & $10 \pm 0.3$ & $12 \pm 0.3$ & \\
\hline
\end{tabular}

Note: P, Petroleum ether; C, Chloroform;A,Acetone; EA, Ethylacetate; E, Ethanol;AQ,Water

The ethanolic extracts of $P$. auriculata demonstrated maximum zone of inhibition $(23 \pm 0.3 \mathrm{~mm})$ against $M$. morganii (Table 2). $P$. auriculata ethanolic extracts was active against all the examined pathogens except $S$. aureus. Compared to all the tested pathogens, $K$. pneumoniae was highly sensitive to all the screened extracts of P. auriculata. The $40-100 \mu \mathrm{g} / \mathrm{ml}$ of ethyl acetate extracts represented more percentage of activity $(18,27,45$ and $63 \%)$ against $S$. pyogenes than the standard amikacin. $100 \mu \mathrm{g} / \mathrm{ml}$ ethanolic extracts represented $18 \%$ and $9 \%$ of more antibacterial activity against $S$. pyogenes and $K$. pneumoniae than the standard amikacin. Similar to ethyl acetate and ethnaolic extracts, acetone extracts also showed more activity against $S$. pyogenes than the standard amikacin.

The significant antibacterial activity was observed in ethanolic extracts compared to other tested extracts of $P$. auriculata. The activity of $P$. auriculata extracts as follows: ethanolic extracts $70 \%>$ petroleum ether $54 \%>$ ethyl acetate $53 \%>$ acetone $48 \%>$ chloroform $34 \%>$ water extract $31 \%$. The ethanolic extracts of $P$. auriculata showed above

Table 3 Antibacterial activity of Plumbago rosea
$50 \%$ activity against Gram positive pathogens and 26\% activity against Gram negative pathogens.

In $P$. rosea, highest activity was observed in ethanolic extracts (91\%) followed by acetone (64\%) and chloroform extracts (59\%). The ethanolic extracts $100 \mu \mathrm{g} / \mathrm{ml}$ showed $45 \%$ higher antibacterial activity against $S$. pyogenes and $10 \%$ more activity against $M$. morganii than the standard amikacin. There was prominent difference on the size of the inhibition zone between ethanolic and other extracts of $P$. rosea. All the screened extracts of $P$. rosea demonstrated the inhibition against $K$. pneumoniae (Table 3 ). Highest antibacterial activity was observed in ethanolic extracts against $B$. subtilis and M. morganii with $22 \pm 0.3$ and $22 \pm 0.7 \mathrm{~mm}$ zone of inhibition. Except $20 \mu \mathrm{g} / \mathrm{ml}$ of water extracts, all other extracts of $P$. rosea with various concentrations (20$100 \mu \mathrm{g} / \mathrm{ml}$ ) showed activity against $K$. pneumonia and maximum zone of inhibition $(20 \pm 0.3 \mathrm{~mm})$ was observed in ethanolic extracts of $P$. rosea. The $100 \mu \mathrm{g} / \mathrm{ml}$ of $P$. rosea ethanolic extracts displayed higher activity against $M$. morganii with $22 \pm 0.5 \mathrm{~mm}$ zone of inhibition.

\begin{tabular}{lllllllll}
\hline \multirow{2}{*}{ Pathogens } & \multicolumn{2}{l}{ Zone of inhibition in $\mathbf{~ m m}$} & & & & & \\
\cline { 2 - 8 } & Extracts Conc. in $\boldsymbol{\mu g}$ & $\mathbf{P}$ & $\mathbf{C}$ & $\mathbf{A}$ & EA & E & AQ & An 30 $\boldsymbol{\mu g}$ \\
\hline & 20 & $7 \pm 0.5$ & nil & $2 \pm 0.5$ & $5 \pm 0.3$ & nil & nil & \\
\multirow{3}{*}{ S. aureus } & 40 & $11 \pm 0.5$ & nil & $9 \pm 0.3$ & $10 \pm 0.3$ & $4 \pm 0.3$ & nil & \\
& 60 & $14 \pm 0.5$ & nil & $10 \pm 0.2$ & $11 \pm 0.3$ & $6 \pm 0.5$ & nil & 24 \\
& 80 & $15 \pm 0.7$ & nil & $12 \pm 0.3$ & $13 \pm 0.3$ & $8 \pm 0.5$ & nil & \\
& 100 & $18 \pm 0.3$ & nil & $14 \pm 0.3$ & $16 \pm 0.3$ & $9 \pm 0.3$ & nil \\
\hline
\end{tabular}




\begin{tabular}{|c|c|c|c|c|c|c|c|c|}
\hline \multirow{2}{*}{ Pathogens } & \multicolumn{8}{|c|}{ Zone of inhibition in $\mathrm{mm}$} \\
\hline & Extracts Conc. in $\mu g$ & $\mathbf{P}$ & C & $\mathbf{A}$ & EA & E & AQ & An $30 \mu g$ \\
\hline \multirow{5}{*}{ B. subtilis } & 20 & $3 \pm 0.3$ & nil & $4 \pm 0.5$ & $6 \pm 0.5$ & $15 \pm 0.3$ & nil & \multirow{5}{*}{25} \\
\hline & 40 & $6.5 \pm 0.7$ & nil & $6 \pm 0.5$ & $8 \pm 0.3$ & $19 \pm 0.3$ & $3 \pm 0.3$ & \\
\hline & 60 & $7 \pm 0.4$ & nil & $11 \pm 0.5$ & $10 \pm 0.4$ & $20 \pm 0.7$ & 4 & \\
\hline & 80 & $1 \mathrm{II} \pm 0.5$ & nil & $14 \pm 0.3$ & $12 \pm 0.3$ & $2 I \pm 0.3$ & $5 \pm 0.3$ & \\
\hline & 100 & $13 \pm 0.3$ & nil & $17 \pm 0.3$ & $14 \pm 0.3$ & $22 \pm 0.3$ & $7 \pm 0.3$ & \\
\hline \multirow{5}{*}{ S. pyogenes } & 20 & nil & nil & $8 \pm 0.3$ & $4 \pm 0.1$ & $3 \pm 0.3$ & nil & \multirow{5}{*}{11} \\
\hline & 40 & nil & $4 \pm 0.3$ & $10 \pm 0.5$ & $7 \pm 0.5$ & $6 \pm 0.3$ & nil & \\
\hline & 60 & nil & $6 \pm 0.5$ & $12 \pm 0.3$ & $8 \pm 0.3$ & $7 \pm 0.5$ & nil & \\
\hline & 80 & nil & $8 \pm 0.3$ & $14 \pm 0.3$ & $12 \pm 0.5$ & $9 \pm 0.5$ & nil & \\
\hline & 100 & nil & $9 \pm 0.5$ & $16 \pm 0.3$ & $13 \pm 0.5$ & $10 \pm 0.5$ & nil & \\
\hline \multirow{5}{*}{ K. pneumoniae } & 20 & $10 \pm 0.5$ & $7 \pm 0.4$ & $8 \pm 0.3$ & $10 \pm 0.3$ & $I I \pm 0.3$ & nil & \multirow{5}{*}{21} \\
\hline & 40 & $13 \pm 0.5$ & $12 \pm 0.3$ & $12 \pm 0.3$ & $12 \pm 0.3$ & $14 \pm 0.3$ & $5 \pm 0.3$ & \\
\hline & 60 & $16 \pm 0.8$ & $14 \pm 0.3$ & $13 \pm 0.5$ & $13 \pm 0.5$ & $16 \pm 0.5$ & $6 \pm 0.3$ & \\
\hline & 80 & $17 \pm 0.4$ & $16 \pm 0.3$ & $15 \pm 0.3$ & $16 \pm 0.3$ & $18 \pm 0.5$ & $6 \pm 0.5$ & \\
\hline & 100 & $19 \pm 0.5$ & $17 \pm 0.3$ & $16 \pm 0.5$ & $18 \pm 0.3$ & $20 \pm 0.5$ & $8 \pm 0.5$ & \\
\hline \multirow{5}{*}{ M. morganii } & 20 & $6 \pm 0.5$ & $9 \pm 0.3$ & nil & nil & $12 \pm 0.3$ & nil & \multirow{5}{*}{20} \\
\hline & 40 & $I I \pm 0.5$ & $12 \pm 0.3$ & nil & nil & $16 \pm 0.3$ & $2 \pm 0.3$ & \\
\hline & 60 & $12 \pm 0.3$ & $14 \pm 0.5$ & nil & nil & $18 \pm 0.3$ & 3 & \\
\hline & 80 & $14 \pm 0.3$ & $16 \pm 0.4$ & nil & nil & $20 \pm 0.3$ & $4 \pm 0.5$ & \\
\hline & 100 & $16 \pm 0.3$ & $18 \pm 0.3$ & nil & nil & $22 \pm 0.7$ & $5 \pm 0.5$ & \\
\hline \multirow{5}{*}{ P. aeruginosa } & 20 & nil & $4 \pm 0.5$ & $8 \pm 0.3$ & $3 \pm 0.3$ & $7 \pm 0.5$ & nil & \multirow{5}{*}{24} \\
\hline & 40 & nil & $9 \pm 0.5$ & $10 \pm 0.3$ & $7 \pm 0.3$ & $9 \pm 0.5$ & nil & \\
\hline & 60 & nil & $10 \pm 0.3$ & $13 \pm 0.3$ & $8 \pm 0.3$ & $13 \pm 0.5$ & nil & \\
\hline & 80 & nil & $13 \pm 0.3$ & $15 \pm 0.2$ & $1 \mathrm{I} \pm 0.5$ & $14 \pm 0.5$ & nil & \\
\hline & 100 & nil & $16 \pm 0.5$ & $18 \pm 0.4$ & $13 \pm 0.4$ & $17 \pm 0.5$ & nil & \\
\hline
\end{tabular}

Note: P, Petroleum ether; C, Chloroform;A,Acetone; EA, Ethylacetate; E, Ethanol;AQ,Water

Similar to other two studied plants, the highest zone of inhibition was observed in ethanolic extracts compared to other tested extracts of P.rosea. The activity of $P$. rosea extracts as follows: ethanolic extracts $91 \%>$ acetone; $64 \%>$ chloroform; $56 \%>$ ethyl acetate; $52 \%>$ petroleum ether; $50 \%>$ water extract. Ethanolic extract of P. rosea showed Gram $99 \%$ activity against positive pathogens and $89 \%$ activity against Gram negative pathogens.

\section{Discussion}

Due to the failure of synthetic drugs, adverse side effects of antibiotics and antibiotic resistance of the microorganisms led to the development of new plant derived antibiotic without any side effects. ${ }^{3,6,25,26}$ Simonsen et al., ${ }^{8}$ documented the use of natural products as new antibacterial drugs. In the present study also we screened the antibacterial potentials of $P$. rosea, $P$. zeylanica and $P$. auriculata against various bacterial pathogens. The ethanolic extract of $P$. zeylanica and $P$. rosea showed high frequency of activity against Gram positive pathogens and Gram negative pathogens. The ethanolic extracts of $P$. auriculata showed leact activity against the tested pathogens. The antibacterial potentials of the P. rosea, P. zeylanica and $P$. auriculata against the tested pathogens may be due to the existence of alkaloids, phenolic substantces. ${ }^{27}$

Antimicrobial activities of Plumbago species have been reported by many workers, Ibrahim et al. ${ }^{17}$ evaluated the antibacterial activity using the methanolic extracts of $P$. indica against $S$. aureus, $S$. typhi, $S$. dysenteriae, B. cereus P. aeruginosa, S. sonnei, V. cholera and E. coli. The highest inhibition was observed against $S$. aureus, E. coli and $S$. typhi compared to other pathogens ranged from 15-27 mm. Devi et al., ${ }^{28}$ studied the antibacterial activity of $P$. zeylanica methanolic leaf, root and stem extract of against Bacillus subtilis. Antibacterial 
activity of methanolic and chloroform extracts of $P$. zeylanica against five different organisms viz., S. pyogenes, S. aureus, Bacillus sp., $P$. aeruginos $a$ and $E$. coli were studied using disc diffusion method. The methanolic extracts were more active against all the tested organisms. ${ }^{18}$ Vishnukanta \& Rana ${ }^{29}$ studied the antibacterial activity of $P$. zeylanica against $S$. gallinarium, E. coli, $P$. vulgaris, $S$ typhimurium, $P$. aeruginosa and $S$. aureus. Among the tested extracts methanolic extract exhibited higher antibacterial activity against all the pathogenic bacteria.

Jetty et al., ${ }^{30}$ evaluated the antimicrobial properties of compounds such as neoisoshinanolone and 1-epineo-isoshinanolone isolated from the roots of P. zeylanica. Among these 1-epineo-isoshinanolone is more active with a MIC of $12.5-25 \mu \mathrm{g} / \mathrm{mL}$ whereas neoisoshinanolone has recorded a MIC of $50-100 \mu \mathrm{g} / \mathrm{mL}$. The activities are compared with plumbagin $[0.78-3.13 \mu \mathrm{g} / \mathrm{mL}]$ and standards streptomycin for bacteria and nystatin for fungi. Jeyachandran et al., ${ }^{20}$ revealed the minimum inhibitory concentration of methanolic, chloroform and aqueous extract of $P$. zeylanica root against E. coli, $S$. typhi and $S$. aureus. The zone of inhibition against $K$. pneumoniae, $S$. marcescens and $B$. subtilis were moderate and lower against Proteus vulgaris and Pseudomonas aeruginosa. The methanolic extract exhibited moderate activity and the aqueous extract weak activity against bacterial strains as assessed by disc diffusion assays.

Rahman \& Anwar ${ }^{21}$ studied the antimicrobial activities of ethanolic extracts of $P$. zeylanica root against 11 human pathogenic bacteria and 6 phytopathogenic fungi using disc diffusion method. $V$. cholerae was found to be the most sensitive. Parekh et al. ${ }^{24}$ studied the antibacterial potential of $P$. zeylanica using agar disc diffusion method and agar well diffusion method against five bacterial strains viz., B. cereus, $S$. aureus, K. pneumoniae, E. coli and P. pseudoalcaligenes. Preliminary screening revealed that methanolic extracts were more potent than the aqueous extracts. Wang \& Huang ${ }^{31}$ revealed the anti-H. pylori activity of ethanolic, ethyl acetate, acetone and aqueous extracts of P. zeylanica.

Paiva et al., ${ }^{14}$ evaluated the antimicrobial activity in the plumbagin isolated from the chloroform extract of $P$. scandens against $S$. aureus, $P$. aeruginosa, $B$. subtilis, $P$. vulgaris and against the yeast $C$. albicans. Plumbagin exhibited relatively specific antimicrobial activity. The growth of $S$. aureus and $C$. albicans was completely inhibited. Antibacterial activity of $P$. zeylanica alcoholic root extracts was studied against multidrug-resistant clinical isolates of bacteria $(S$. paratyphi, S. aureus, E. coli, S. dysenteriae). The extracts exhibited strong antibacterial activity against all test bacteria irrespective of their antibiotic resistance behaviour. ${ }^{9}$ Except Wang \& Hang et al., ${ }^{31}$ observation, all others were recorded the highest frequency of antibacterial activity in the methanolic extracts of Plumbago species. Tharmaraj \& Antonysamy ${ }^{23}$ observed the antibacterial activity of $P$. rosea against $K$. pneumoniae, $B$. subtilis, $S$. aureus, $P$. aeruginosa and $P$. vulgaris with maximum zone inhibition $20,19,17,16$ and $16 \mathrm{~mm}$ respectively. In the present study we observed better results then the previous observations. $100 \mu \mathrm{g}$ of ethanolic extracts of $P$. rosea showed maximum zone of inhibition against $B$. subtilis and $K$. pneumoniae with 22 and $20 \mathrm{~mm}$ respectively. $100 \mu \mathrm{g}$ of acetone extracts of $P$. rosea demonstrated maximum zone $(18 \mathrm{~mm})$ of inhibition against $P$. aeruginosa earlier $16 \mathrm{~mm}$ of inihibition was observed with $250 \mu \mathrm{g}$ of methanolic extracts. In addition, the ethanolic and acetone extracts of $P$. rosea illustrated the inhibition against $M$. morganii $(22 \mathrm{~mm})$ and $S$. pyogenes $(16 \mathrm{~mm})$ respectively.
Similar to that in the present study also we observed the high frequency of antibacterial activity in the ethanolic extracts of three Plumbago species. The antibacterial action of various aerial parts extracts of Plumbago species may indicate their potential as antibacterial herbal remedies. Further work is needed to locate the active principle from the various extracts and their phyto pharmaceutical studies. Research into the effects of local medicinal plants is expected to boost the use of these plants in the therapy against disease caused by the test bacterial species and other microorganisms.

\section{Acknowledgments}

None.

\section{Conflicts of interest}

Authors declare that there is no conflict of interest.

\section{References}

1. Clement MV. Chemopreventive agent resveratrol, a natural product derived from graps, triggers CD 95 Signaling- dependent apoptosis in human tumor cells. Blood. 1998;92(3):996-1002.

2. Evans CE, Banso A, Samuel OA. Efficacy of some nupe medicinal plants against Salmonella typhi: an in vitro study. J Ethnopharmacol. 2002;80(1):21-24

3. Vijaya K, Ananthan S. Microbiological screening of Indian medicinal plants with special reference to enteropathogens. J Altern Complement Med. 1997;3(1):13-20.

4. Dilhuydy JM. Patients attraction to complementary and alternative medicine (CAM): a reality which physicians can neither ignore nor deny. Bull Cancer. 2003;90(7):623-628.

5. Pretorius CJ, Watt E. Purification and identification of active components of Carpobrotus edulis L. J Ethnopharmarcol. 2001;76(1):87-91.

6. Sharif MDM, Banik GR. Status and utilization of medicinal plants in Rangamati of Bangladesh. Res J Agric Biol Sci. 2006;2(6):268-273.

7. Dorni AIC, Vidyalakshmi KS, Hannah RV, et al. Antiinflammatory activity of Plumbago capensis. Pharmacognosy magazine. 2006;2:239-243.

8. Simonsen HT, Nordskjold JB, Smitt UW, et al. In vitro screening of Indian medicinal plants for antiplasmodial activity. $J$ Ethnopharmacol. 2001;74(2):195-204

9. Ahmad I, Mehmood Z, Mohammad F, et al. Antimicrobial potency and synergistic activity of five traditionally used Indian medicinal plants. Journal of Medicinal and Aromatic Plant Sciences. 20002;23:173-176.

10. Mehmood Z, Ahmad I, Mohammad F, et al. Indian medicinal plants: A potential source of anti candidal drugs. Pharmaceutical Biology. 1999;37(3):237-242.

11. Oyedapo OO. Studies on the bioactivity of the extract of Plumbago zeylanica. International Journal of Pharmacognosy. 1996;34(5):346-348.

12. Olagunju JA, Jobi AA, Oyedapo OO. An investigation into the biochemical basis of the observed hyper glycaemia in rats treated with ethanol root: An update. International Journal of Pharmacology. $1999 ; 7: 316-324$

13. Sharma Anubhuti, Sharma Pratibha. Genetic and Phytochemical analysis of Cluster bean (Cyamopsis tetragonaloba (L.) Taub) by RAPD and HPLC. Journal of Recent Research Science. 2013;2(2):1-9.

14. Paiva SR, Marques SS, Figueiredo MR, et al. Plumbaginale: A pharmacology approach. Floresta e Ambiente. 2003;10:98-105. 
15. Gangopadhyay M, Dewanjee S, Chakraborty D, et al. Role of exogenous phytohormones on growth and plumbagin accumulation in Plumbago indica hairy roots and conservation of elite root clones via synthetic seeds. Indian Journal of Crops Production. 2011;33:445-450.

16. Dinda B, Hajra AK, Chel G. 1,4, Naphthoquinonens of Plumbago sp. A review. Journal of Indian Chemical Society. 1997;74:974-979.

17. Ibrahim M, Baura J, Ahasan Q, et al. Preliminary Phytochemical and Pharmacological Investigations of Alpinia conchigera Griff. and Plumbago indica L. Bangladesh Pharmaceutical Journal. 2012;15(2):153-157.

18. Devi SM, Thenmozhi M. Antibacterial Activity of Plumbago zeylanica Leaf Extract. International Journal of Research in Biomedicine and Biotechnology. 2011;1(1):1-4.

19. Vishnukanta S, Rana AC. Evaluation of anticonvulasant activity Plumbago zeylanica Linn leaf extract. Asian Journal of Pharmaceutical Clinical Research. 2011;3(1):76-78.

20. Jeyachandran R, Mahesh A, Cindrella, et al. Antibacterial activity of plumbagin and root extracts of Plumbago zeylanica L. Acta Biologica Cracoviensia series botanica. 2009;51(1):17-22.

21. Rahman MS, Anwar MN. Antimicrobial activity of crude extract obtained from the root of Plumbago zeylanica. Bangladesh Journal of Microbiology. 2007;24(1):73-75.

22. Parekh J, Chanda S. In vitro antibacterial activity of the crude methanol extract of Woodfordia fruticosa Kurz. Flower (Lythaceae). Brazilian Journal of Microbiology. 2007;38:204-207.

23. Tharmaraj RJJM, Antonysamy JM. Phytochemical and Bio-efficacy
Studies on Plumbago rosea L. In: Parimelazhagan T (Ed.), Scientific Basis of Herbal Medicine. Daya Publishing House Astral International Pvt Ltd: New Delhi, India; 2013. 95-100 p.

24. Parekh J, Chanda SV. In vitro Antimicrobial activity and Phytochemical analysis of some Indian medicinal plants. Turk J Biol. 2007;31:53-58.

25. Cragg GM, Newman DJ. Medicinals for the millennia: the historical record. Ann N Y Acad Sci. 2001;953:3-25.

26. Hostettmann K. Assays for bioactivity: Methods in Plant Biochemistry. Academic Press: San Diego, USA; 1991.

27. Tharmaraj RJJM, Antonysamy JM. Studies on Inter-specific Variation in the Genus Plumbago (Plumbaginaceae) from South India using Phytochemical Analysis. Indo American Journal of Pharm Research. 2013;3(5):3892-3902.

28. Devi VG, Vijayan C, John A, et al. Pharmacognostic and antioxidant studies on Clerodendrum inerme and identification of ursolic acid as marker compound. International Journal of Pharmacy \& Pharmaceutical Sciences. 2012;4(2):145-148.

29. Vishnukanta S, Rana AC. Evaluation of anti convulasant activity Plumbago zeylanica Linn leaf extract. Asian Journal of Pharmaceutical Clinical Research. 2011;3(1):76-78.

30. Jetty A, Subhakar C, Rajagopal D, et al. Antimicrobial activities of neoand 1- epineo-isoshinanolones from Plumbago zeylanica roots. Pharm Biol. 2010;48(9):1007-1011.

31. Wang YC, Huang TL. Anti-Helicobacter pylori activity of Plumbago zeylanica L. FEMS Immunol Med Microbiol. 2005;43(3):407-412. 